\title{
Implication of dysregulation of the canonical wingless-type MMTV integration site (WNT) pathway in diabetic nephropathy
}

\author{
T. Zhou $\cdot$ X. He $\cdot$ R. Cheng $\cdot$ B. Zhang $\cdot$ R. R. Zhang $\cdot$ \\ Y. Chen • Y. Takahashi • A. R. Murray • K. Lee • \\ G. Gao $\cdot$ J-x. Ma
}

Received: 27 March 2011 / Accepted: 2 August 2011 / Published online: 21 October 2011

(C) Springer-Verlag 2011

\begin{abstract}
Aims/hypothesis The wingless-type MMTV integration site (WNT) pathway mediates multiple physiological and pathological processes, such as inflammation, angiogenesis and fibrosis. The aim of this study was to investigate whether
\end{abstract}

Electronic supplementary material The online version of this article (doi:10.1007/s00125-011-2314-2) contains peer-reviewed but unedited supplementary material, which is available to authorised users.

T. Zhou • G. Gao

Department of Biochemistry, Zhongshan Medical School,

Sun Yat-sen University,

Guangzhou, People's Republic of China

T. Zhou $\cdot$ X. He $\cdot$ R. Cheng $\cdot$ Y. Chen $\cdot$ A. R. Murray $\cdot$ J-x. Ma

Department of Physiology,

University of Oklahoma Health Sciences Center,

Oklahoma City, OK, USA

B. Zhang $\cdot$ K. Lee

Department of Cell Biology,

University of Oklahoma Health Sciences Center,

Oklahoma City, OK, USA

\section{J-x. Ma $(\triangle)$}

Harold Hamm Oklahoma Diabetes Center,

941 Stanton L. Young Blvd, BSEB 328B,

Oklahoma City, OK 73104, USA

e-mail: jian-xing-ma@ouhsc.edu

\section{Y. Takahashi}

Department of Medicine Endocrinology,

University of Oklahoma Health Sciences Center,

Oklahoma City, OK, USA

\section{R. R. Zhang}

Department of Pathology,

University of Oklahoma Health Sciences Center,

Oklahoma City, OK, USA canonical WNT signalling plays a role in the pathogenesis of diabetic nephropathy.

Methods Expression of WNT ligands and frizzled receptors in the canonical WNT pathway in the kidney was compared at the mRNA level using real-time RT-PCR between Akita mice, streptozotocin-induced diabetic rats and $d b / d b$ mice and their respective non-diabetic controls. Renal function was evaluated by measuring the urine albumin excretion. Human renal proximal tubular epithelial cells were treated with high-glucose medium and 4-hydroxynonenal (HNE). Levels of $\beta$-catenin, connective tissue growth factor and fibronectin were determined by western blot analysis.

Results Some of the WNT ligands and frizzled receptors showed increased mRNA levels in the kidneys of Akita mice, streptozotocin-induced diabetic rats and $d b / d b$ mice compared with their non-diabetic controls. Renal levels of $\beta$-catenin and WNT proteins were upregulated in these diabetic models. Lowering the blood glucose levels by insulin attenuated the activation of WNT signalling in the kidneys of Akita mice. In cultured human renal proximal tubular epithelial cells, both high glucose and HNE activated WNT signalling. Inhibition of WNT signalling with a monoclonal antibody blocking LDL-receptor-related protein 6 ameliorated renal inflammation and fibrosis and reduced proteinuria in Akita mice.

Conclusions/interpretation The WNT pathway is activated in the kidneys of models of both type 1 and 2 diabetes. Dysregulation of the WNT pathway in diabetes represents a new pathogenic mechanism of diabetic nephropathy and renders a new therapeutic target.

Keywords Diabetic nephropathy · Fibrosis · Insulin · Kidney $\cdot$ Oxidative stress $\cdot$ WNT 


\begin{tabular}{|c|c|}
\hline \multicolumn{2}{|c|}{ Abbreviations } \\
\hline Ad- $\beta$-gal & Adenovirus expressing $\beta$-galactosidase \\
\hline Ad-S37A & $\begin{array}{l}\text { Adenovirus expressing a constitutively } \\
\text { active mutant of } \beta \text {-catenin }\end{array}$ \\
\hline CTGF & Connective tissue growth factor \\
\hline FZD & Frizzled \\
\hline HNE & 4-Hydroxynonenal \\
\hline HRPTC & $\begin{array}{l}\text { Human renal proximal tubular epithelial } \\
\text { cells }\end{array}$ \\
\hline ICAM-1 & Intercellular adhesion molecule 1 \\
\hline LCM & Plain L cell conditioned medium \\
\hline LRP & LDL-receptor-related protein \\
\hline STZ & Streptozotocin \\
\hline VEGF & Vascular endothelial growth factor \\
\hline WCM & WNT3A-conditioned medium \\
\hline WNT & Wingless-type MMTV integration site \\
\hline WT & Wild-type \\
\hline
\end{tabular}

\section{Introduction}

Diabetic nephropathy, one of the most common diabetic complications, is the leading cause of end-stage renal disease, and its prevalence is increasing worldwide [1]. The renal lesions in type 1 and type 2 diabetes are similar [2], and involve multiple cell types, including glomerular podocytes, mesangial cells and tubular epithelial cells [3]. Although the glomerulus has been the focus of intensive investigation, recently tubulo-interstitial injury has drawn more attention and interest in diabetic nephropathy research $[4,5]$.

Hyperglycaemia-induced AGE, cytokines, chemokines, growth factors and oxidative stress have been shown to mediate renal damage in human and experimental diabetes [1, 6]. Hyperglycaemia works through both metabolic and haemodynamic mediators to activate multiple signalling pathways and transcription factors, resulting in renal inflammation, angiogenesis, extracellular matrix accumulation/fibrosis, apoptosis/ nephrin loss, proteinuria, hyperfiltration and eventual renal insufficiency [3].

The wingless-type MMTV integration site (WNT) signalling pathway is a multifunctional pathway that regulates cell proliferation and differentiation, stem cell maintenance, angiogenesis, inflammation, fibrosis and carcinogenesis [7]. WNT ligands are secreted cysteine-rich glycosylated proteins that bind to a receptor complex comprised of frizzled (FZD) receptors and LDL-receptor-related protein (LRP) 5 or 6. Activated LRP5 or LRP6 transduces the signal from extracellular ligands to the intracellular cascade and leads to inactivation of the 'destructive complex', which is composed of glycogen synthase kinase-3 $\beta$ (GSK3 $\beta$ ), axin and adenomatous polyposis coli. Inactivation of the 'destructive complex' prevents the proteasomal degradation of the transcription factor $\beta$-catenin and promotes its accumulation and nuclear translocation. Once $\beta$-catenin translocates into the nucleus, it dimerises with $\mathrm{T}$ cell factor (TCF) and regulates transcription of WNT target genes, including those encoding cyclin D1, vascular endothelial growth factor (VEGF), c-Myc and connective tissue growth factor (CTGF) [8-10].

Our previous studies have shown that the WNT pathway is overactivated in retinas from human patients with diabetic retinopathy and in those from animal models of diabetes [11]. Furthermore, blockage of WNT signalling attenuates retinal inflammation and neovascularisation in diabetic retinopathy models [11]. Documented studies suggest that the presence of either retinopathy or nephropathy may contribute to the development of the other, especially in type 1 diabetic patients [12]. More importantly, WNT signalling has been shown to be a key regulator of kidney development [13] and has been implicated in certain kidney diseases [14-16]. It is reasonable to hypothesise that the WNT signalling pathway is implicated in the pathogenesis of diabetic nephropathy.

The present study investigates the pathogenic role of the canonical WNT pathway in diabetic nephropathy and identifies the cause for the activation of the WNT pathway in the kidneys of experimental diabetic models.

\section{Methods}

Materials and antibodies L-glucose and D-glucose were purchased from Sigma (St. Louis, MO, USA), and 4hydroxynonenal (HNE) was purchased from Calbiochem (Madison, WI, USA). Antibodies against $\beta$-catenin, cyclin D1, fibronectin and CTGF were from Santa Cruz Biotechnology (Santa Cruz, CA, USA). The antibodies against WNT3A and c-Myc were from Cell Signaling (Danvers, MA, USA). Antibodies against $\beta$-actin and horseradish peroxidase-conjugated secondary antibodies were obtained from Abcam (Cambridge, MA, USA). Conditioned media containing WNT3A were prepared from mouse L cells stably producing WNT3A (ATCC, Manassas, VA, USA). Control conditioned media were obtained from parental L cells (ATCC).

Animal models of diabetes Male Akita mice and $d b / d b$ mice were purchased from The Jackson Laboratory (Bar Harbor, ME, USA) and female Brown Norway rats were purchased from Harlan (Indianapolis, IN, USA). Care, use and treatment of all animals in this study were in strict agreement with the Guidelines for the Care and Use of Laboratory Animals set forth by the University of Oklahoma. Experimental diabetes was induced in the rats as described by Chen et al. [11]. 
Insulin treatment Akita mice (blood glucose $\geq 19.5 \mathrm{mmol} / \mathrm{l}$ ) were implanted with subcutaneous insulin pellets (LinShin Canada, Toronto, ON, Canada), according to the manufacturer's protocol, to lower blood glucose levels for 8 weeks. Blood glucose levels were monitored weekly. Animals with poor glycaemic control received additional insulin pellets.

Antibody preparation Monoclonal antibody 2F1 was raised using a recombinant peptide comprising the E1E2 domain of LRP6. The antibody was purified from a hybridoma cell line by affinity chromatography using a protein $G$ column (Thermo Scientific, Waltham, MA, USA) and from the ascites fluid of BALB/c mice injected with the $2 \mathrm{~F} 1$ hybridoma cells. The purified antibody was dialysed against PBS, sterile filtered and administrated intraperitoneally. Non-specific mouse IgG purchased from Vector Laboratories (Burlingame, CA, USA) was subjected to the same procedure.

Real-time PCR Total RNA was isolated from kidneys using TRIzol according to the manufacturer's protocol (Invitrogen, Carlsbad, CA, USA). The cDNA was reverse transcribed using a reverse transcription reagent kit (Applied Biosystems, Foster City, CA, USA) and quantitative RTPCR was conducted as described by Wang et al. [17].

Cell culture Primary human renal proximal tubular epithelial cells (HRPTC) were purchased from ATCC and cultured according to the protocol recommended by ATCC.

Subcellular fractionation Kidney tissue and cells were fractionated using FractPrep (BioVision, Mountain View, CA, USA) following the manufacturer's protocol.

Western blot analysis Western blot analysis was performed as described by Zhou et al. [18].

Immunostaining Immunocytochemistry was performed as described by Zhou et al. [18]. Immunohistochemistry was performed as described by Chen et al. [11]. Briefly, paraffin-embedded kidney sections were stained with the antibody for $\beta$-catenin at a dilution of 1:250 for $1 \mathrm{~h}$. After thorough washes with PBS, immunosignals were developed using an $\mathrm{ABC}$ kit (Vectastain $\mathrm{ABC}$; Vector Laboratories) according to the manufacturer's protocol.

Measurement of microalbuminuria and creatinine Mice were kept in individual metabolism cages. A $24 \mathrm{~h}$ urine sample was collected from each mouse. Urine albumin was measured by ELISA according to the manufacturer's protocol (Exocell, Philadelphia, PA, USA). The total amount of albumin in the $24 \mathrm{~h}$ urine was calculated accordingly. Urine creatinine was measured by HPLC according to the protocol from the Animal Models of Diabetic Complications Consortium [19].

ELISA for fibronectin The kidneys were dissected, homogenised and centrifuged at $800 \mathrm{~g}$ for $5 \mathrm{~min}$. The total protein concentration in the supernatant fraction was measured using the bicinchoninic acid protein assay reagent kit (Pierce, Rockford, IL, USA). A commercial mouse fibronectin ELISA kit (Assaypro, St Charles, MO, USA) was used to measure fibronectin levels according to the manufacturer's instructions and normalised by total protein concentrations in the kidney.

Statistical analysis Data are presented as mean \pm SD. Comparisons were performed by two-tailed Student's $t$ test. A difference of $p<0.05$ was considered statistically significant.

\section{Results}

Activation of the canonical WNT pathway in the kidneys of Akita mice Akita mice, a genetic model of type 1 diabetes, are commonly used for studies of diabetic nephropathy as they develop severe albuminuria and consistent renal pathological changes [20]. As WNT signalling regulates inflammation and fibrosis, we examined if the WNT pathway is activated in the kidneys of Akita mice. We first performed a comprehensive analysis of the expression of the nine WNT ligands and ten FZD receptors involved in the canonical WNT pathway by real-time RT-PCR. As shown in Fig. 1a, the expression of most of the genes encoding WNT ligands of the canonical WNT pathway, with the exceptions of $W n t 2$ and $W n t 9 a$, were significantly upregulated in the kidneys of 9-month-old Akita mice compared with the age-matched wild-type (WT) controls. Among FZD genes analysed, however, only $F z d 9$ showed a significantly increased mRNA level in the kidneys of the Akita mice, while expression of the other receptor genes was not changed (Fig. 1b).

To confirm the upregulation of WNT ligands in the kidney, we have compared the protein levels of WNT3A, a commonly studied ligand in the canonical WNT pathway. As shown by western blot analysis, levels of WNT3A protein were also increased in the kidneys of Akita mice compared with those in the non-diabetic control mice (Fig. 1c, d). Further, to determine if the downstream WNT pathway is indeed activated, we examined the accumulation and nuclear translocation of $\beta$ catenin, a key effector of WNT signalling. As shown in Fig. 1e, f, both the cytosolic and nuclear $\beta$-catenin levels were increased significantly in the Akita kidneys, indicating the activation of $\beta$-catenin in the diabetic kidney. 
Fig. 1 Activation of the canonical WNT pathway in the kidneys of Akita mice. Realtime PCR analysis of mRNA levels of WNT ligands (a) and FZD receptors (b) in the kidneys of WT and Akita mice. Values are mean $\pm \mathrm{SD}, n=3$ in WT (white bars), $n=6$ in Akita mice (black bars); ${ }^{*} p<0.05$ and ${ }^{* *} p<$ 0.01 . Representative western blots (c, e, g) and quantification by densitometry $(\mathbf{d}, \mathbf{f}, \mathbf{h})$ show increased protein levels of WNT3A (c, d), cytosolic and nuclear levels of $\beta$-catenin (e, f) and protein levels of WNT target genes $(\mathbf{g}, \mathbf{h})$ in the kidneys of Akita mice. GAPDH, glyceraldehyde-3-phosphate dehydrogenase. Each lane represents an individual animal. Values are mean $\pm \mathrm{SD}, n=3$; WT, white bars; Akita mice, black bars; $* p<0.05$ and $* * p<0.01$
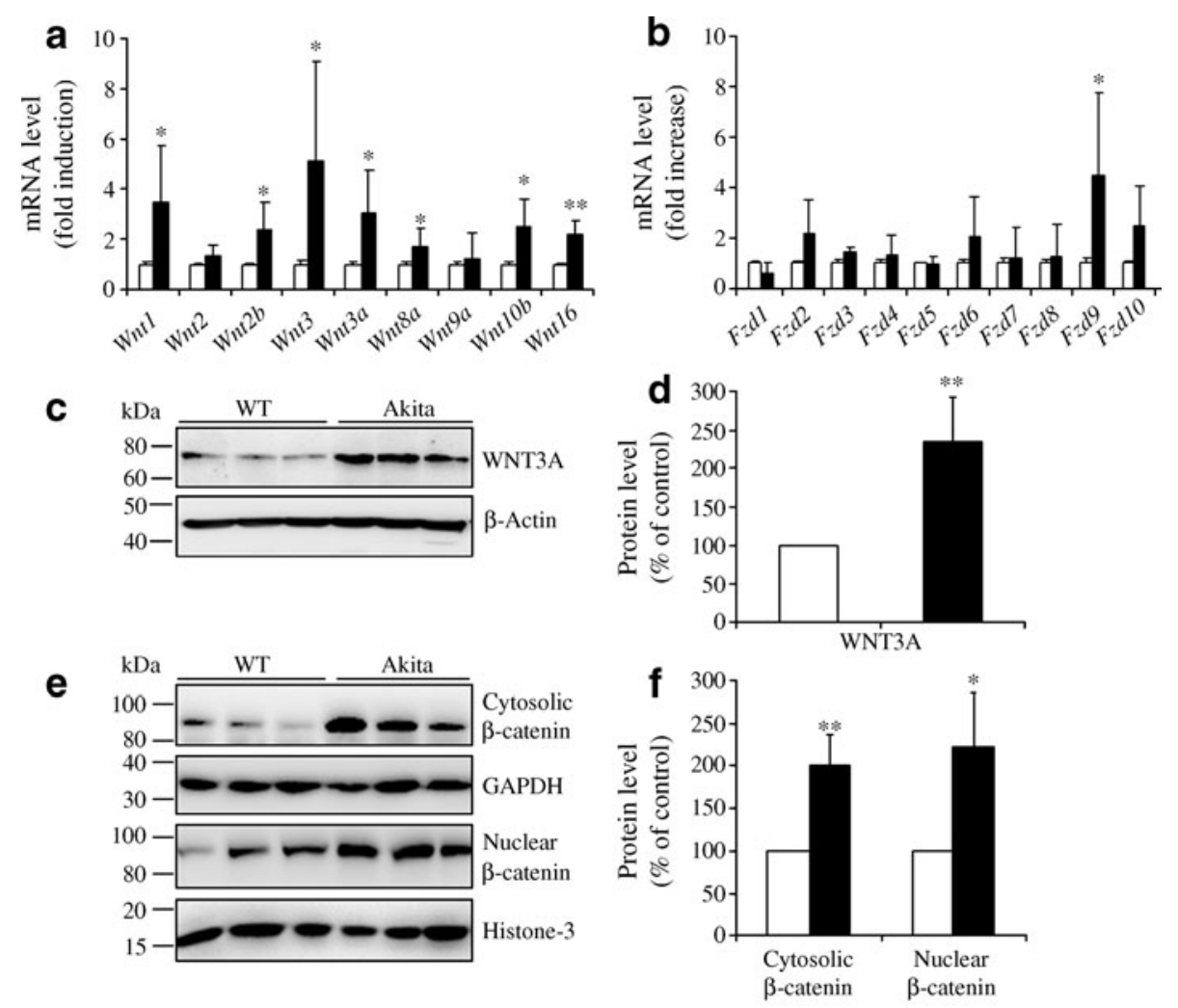

g

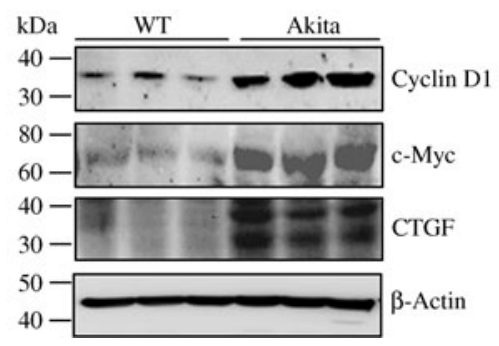

We also studied the production of proteins encoded by several target genes of WNT signalling in the diabetic kidney. As shown in Fig. 1g, h, the protein levels of products of known WNT target genes, including cyclin D1, c-Myc and CTGF, were increased in the Akita mouse kidney compared with that of the WT control. These results demonstrate activation of the WNT pathway in diabetic nephropathy. Moreover, we performed immunohistochemistry to identify the localisation of $\beta$-catenin in the kidney. Immunostaining of the kidney sections using an antibody specific for $\beta$ catenin showed that the Akita mouse kidney has a more intensive $\beta$-catenin signal in the renal tubules and interstitium compared with that in the WT mouse kidney (Fig. 2ad). In addition, increased $\beta$-catenin signals were detected in the nuclei of tubular cells, suggesting nuclear translocation of $\beta$-catenin in the diabetic kidney (Fig. 2d, e).

Activation of the canonical WNT pathway in the kidney of streptozotocin-induced diabetic rats We also determined the activation of the canonical WNT pathway in the kidneys of diabetic rats using streptozotocin (STZ)-induced diabetes as a model. Real-time RT-PCR analysis showed that, similar to the Akita mice, the mRNA levels of Wnt3, $W n t 3 a$ and $W n t 10 b$ were increased in the kidneys of STZinduced diabetic rats at 12 weeks after the onset of diabetes compared with the non-diabetic rats. However, the expression of other WNT ligand genes was not significantly changed (Fig. 3a). The mRNA levels of Fzd1, Fzd6 and $F z d 10$ were significantly upregulated in the diabetic kidney compared with the non-diabetic controls (Fig. 3b). Consistently, renal levels of WNT3A protein were increased in the STZ-induced diabetic rats (Fig. 3c, d). Further, both the cytosolic and nuclear $\beta$-catenin levels were increased significantly in the diabetic kidneys compared with those in the control kidneys (Fig. 3e, f). Moreover, the expression of genes encoding c-Myc and cyclin D1 were upregulated in the diabetic kidneys (Fig. 3g), suggesting activation of the canonical WNT pathway. 


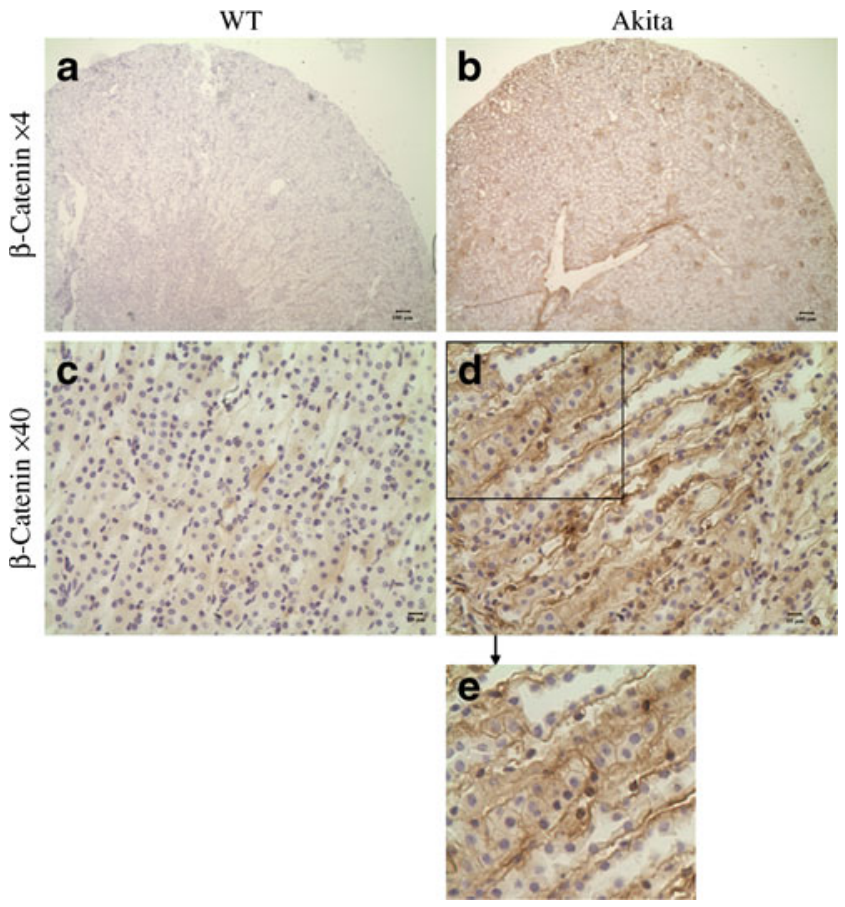

Fig. 2 The location of $\beta$-catenin in Akita mouse kidney. Kidney sections from WT $(\mathbf{a}, \mathbf{c})$ and Akita $(\mathbf{b}, \mathbf{d})$ mice at the age of 9 months were immunostained with an antibody for $\beta$-catenin using DAB staining (brown colour). Images are representatives of three individual animals in each group. Scale bar $100 \mu \mathrm{m}$, magnification $\times 4(\mathbf{a}, \mathbf{b})$, scale bar $10 \mu \mathrm{m}$, magnification $\times 40(\mathbf{c}, \mathbf{d})$. e High-magnification image from the boxed area of $\mathbf{d}$

Activation of the canonical WNT pathway in the kidney of $\mathrm{db} / \mathrm{db}$ mice In order to determine if activation of the WNT pathway also occurs in the kidneys of mouse models of type 2 diabetes, we evaluated WNT signalling activation in the renal tissue of $d b / d b$ mice, a genetic model. As shown by real-time RT-PCR, mRNA levels of a variety of WNT ligands were upregulated in the $d b / d b$ mouse kidney, similar to the results with Akita mice (Fig. 4a). Among the FZD receptors analysed by real-time RT-PCR, Fzd4 mRNA levels were significantly elevated in the kidneys of $d b / d b$ mice (Fig. 4b). Similar to findings with the Akita mice, the expression of Wnt3a was upregulated both at the mRNA and protein levels in the kidneys of $d b / d b$ mice (Fig. 4c, d).

Next, we examined $\beta$-catenin activation in the $d b / d b$ kidney. Western blot analysis showed that the cytosolic and nuclear levels of $\beta$-catenin were both increased in the $d b / d b$ mouse kidney compared with the age-matched non-diabetic controls (Fig. 4e, f), demonstrating the accumulation and nuclear translocation of $\beta$-catenin. Furthermore, renal levels of WNT target genes for cyclin D1, c-Myc and CTGF were increased in $d b / d b$ mice compared with controls (Fig. 4g, h), reflecting activation of the WNT signalling in the kidneys of $d b / d b$ mice.
Insulin treatment attenuated the activation of the canonical WNT pathway in the Akita mouse kidney To establish the potential causative role of hyperglycaemia in the activation of the canonical WNT pathway in the kidney of the diabetic animal models, we administered insulin to Akita mice for 8 weeks. Consistent with the findings shown in Fig. 1, protein levels of WNT3A, $\beta$-catenin, c-Myc and CTGF were significantly increased in the kidneys of Akita mice without insulin treatment, whereas insulin treatment attenuated the increase of these proteins, correlating with the reduced blood glucose levels in the same mice (Fig. 5a-c). This suggests that hyperglycaemia plays a causative role in the activation of WNT signalling in diabetic kidneys.

Activation of the WNT pathway by high glucose and HNE in HRPTC HRPTC are the primary target of tubulo-interstitial pathologies in diabetic nephropathy [21, 22]. In addition to hyperglycaemia, oxidative stress has been shown to play an important role in diabetic nephropathy. Increased levels of HNE in the plasma and lymphocytes were found in type 2 diabetic patients with nephropathy [23] and accumulation of HNE protein adducts was detected in STZ-induced animal models of diabetes [24]. Therefore, we evaluated the direct effects of high-glucose medium and HNE on WNT signalling in HRPTC. As shown in Fig. 6, high-glucose medium elevated cytosolic $\beta$-catenin levels and production of fibronectin in HRPTC. Similarly, HNE treatment also upregulated cytosolic and nuclear levels of $\beta$-catenin. These results suggested that the effect of high glucose concentration and lipid peroxidation can directly activate the canonical WNT pathway in renal tubular cells.

Activation of WNT signalling alone induced fibrogenic factors in HRPTC It has been reported that HRPTC are the primary cells that respond to WNT activation after kidney injury [25]. As hyperglycaemia and $\mathrm{HNE}$ can trigger multiple signalling pathways [22, 26-29], in order to confirm the direct effect of WNT activation on renal fibrogenesis in diabetic nephropathy, primary HRPTC were exposed to WNT3A-conditioned medium (WCM); plain L cell conditioned medium (LCM) was used as a control. As shown by western blot analysis, WCM increased cytosolic and nuclear $\beta$-catenin levels significantly compared with LCM (Fig. 7ad). Immunocytochemistry revealed that $\beta$-catenin was distributed mainly in the membrane pool and cytosol, and was undetectable in the nuclei of LCM-treated control cells (Fig. 7e, g). WNT3A induced an apparent increase of $\beta$ catenin in the nuclei (Fig. 7f, h), indicating activation of the canonical WNT pathway by WNT3A. In the same cells, CTGF and fibronectin levels were significantly elevated by WCM compared with those treated with LCM (Fig. 7i, j), suggesting that activation of WNT signalling alone can induce a fibrogenic response in proximal tubular cells. 
Fig. 3 Activation of the WNT pathway in the kidneys of STZinduced diabetic rats. Real-time PCR analysis of the mRNA levels of WNT ligands (a) and FZD receptors (b) in the kidneys of non-diabetic rats and STZinduced diabetic rats at 12 weeks following the onset of diabetes. Values are mean $\pm \mathrm{SD}, n=5$ in non-diabetic (white bars), $n=6$ in STZ-induced diabetic rats (black bars); ${ }^{*} p<0.05$ and ${ }^{* *} p<0.01$. c-f Representative western blots (c, e) and quantification with densitometry $(\mathbf{d}, \mathbf{f})$ show increased protein levels of WNT3A (c, d) and cytosolic and nuclear levels of $\beta$-catenin $(\mathbf{e}, \mathbf{f})$ in the kidneys of STZ-induced diabetic rats. GAPDH, glyceraldehyde-3-phosphate dehydrogenase; TBP, TATA box-binding protein. Each lane represents an individual animal. Values are mean $\pm \mathrm{SD}, n=3$; non-diabetic, white bars; STZ-induced diabetic rats, black bars; ${ }^{*} p<0.05$ and $*_{*} p<0.01$. g Real-time PCR analysis of mRNA levels of Ccnd1 (encoding cyclin D1) and $c-M y c$ in the kidneys of nondiabetic and diabetic rats. Values are mean $\pm \mathrm{SD} ; n=5$ for nondiabetic (white bars), $n=6$ for STZ-diabetic rats (black bars); ${ }^{*} p<0.05$ and $* *_{p}<0.01$. NonDM, non-diabetic rats; STZ-DM, STZ-induced diabetic rats
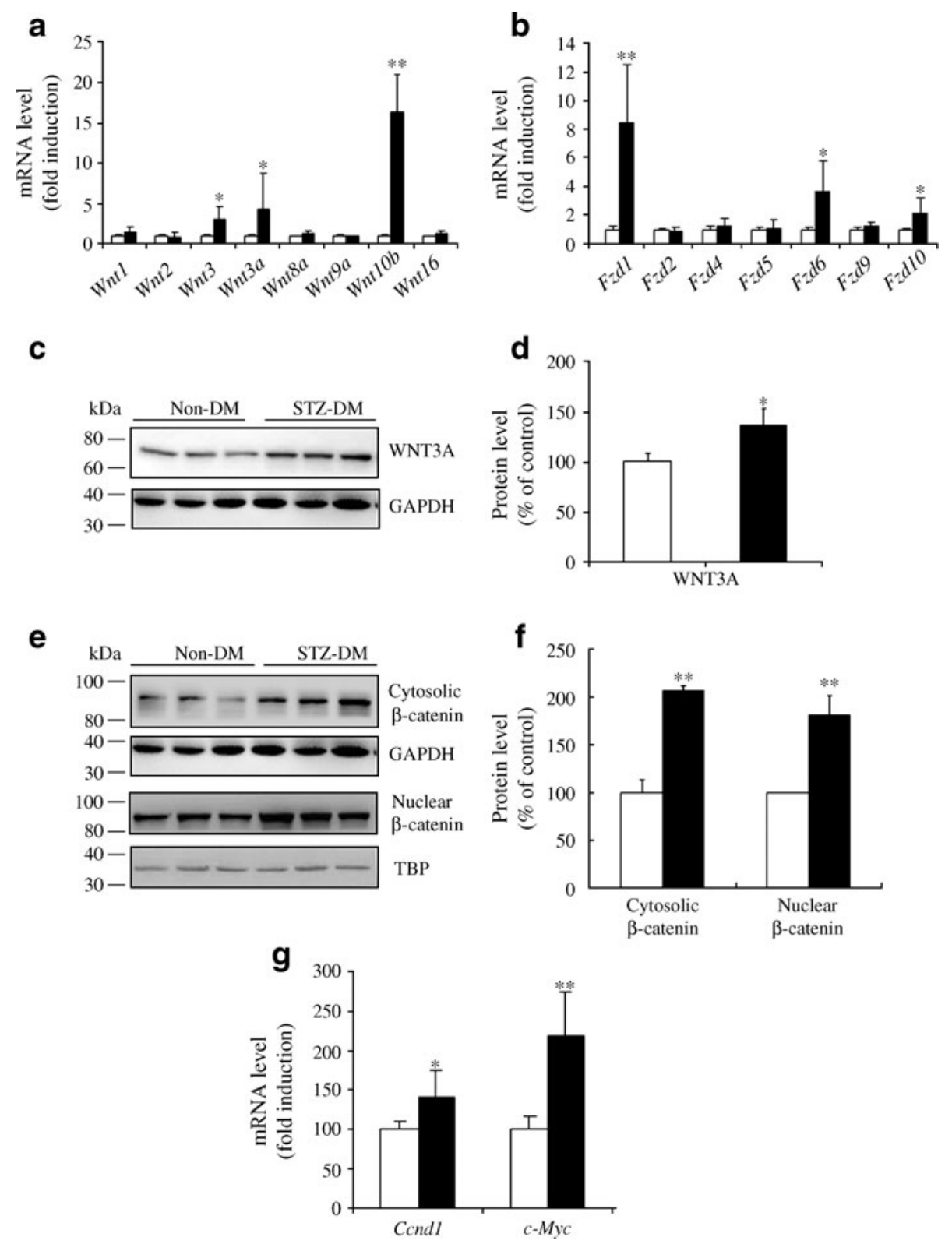

To confirm the effect of WNT signalling on fibrogenesis, an adenovirus expressing a constitutively active mutant of $\beta$-catenin (Ad-S37A), in which the phosphorylation site S37 was substituted by Ala (S37A), was used. Primary HRPTC were infected with Ad-S37A and with adenovirus expressing $\beta$-galactosidase (Ad- $\beta$-gal) at the same multiplicity of infection (MOI) as control. At $48 \mathrm{~h}$ following viral infection, cytosolic and nuclear levels of $\beta$-catenin were increased significantly in cells infected with Ad-S37A compared with those infected with Ad- $\beta$-gal (Fig. 8a-d). Similarly, Ad-S37A induced an apparent increase of $\beta$-catenin signal in the nuclei (Fig. 8e-h). In addition, the production of fibronectin was upregulated significantly in the cells infected with Ad-S37A (Fig. 8i, j). Together with the observations in Fig. 6, these results suggest that activation of the WNT pathway contributes to the overproduction of pro-fibrogenic factors in diabetic nephropathy.

Antagonists of the WNT signalling pathway ameliorate diabetic nephropathy in Akita mice To further establish the pathogenic role of overactivation of WNT signalling in diabetic nephropathy, we examined the effects of a specific blocker of the canonical WNT pathway. We injected 2F1 antibody, which binds to the ligand-binding domain of LRP6 and blocks WNT pathway activation [30], into Akita mice; the same dose of non-specific mouse IgG was used as the negative control. Western blot analysis showed that the accumulation of $\beta$-catenin in the kidneys of Akita mice was 
Fig. 4 Activation of the canonical WNT pathway in the kidney of $d b / d b$ mice. Real-time PCR analysis of mRNA levels of WNT ligands (a) and FZD receptors (b) in the kidneys of WT and $d b / d b$ mice at the age of 6 months. Values are mean \pm SD; $n=3$ for WT (white bars); $n=6$ for $d b / d b$ mice (black bars); ${ }^{*} p<0.05$ and $* * p<0.01$.

c-h Representative western blots (c, e, g) and densitometry analyses (d, f, h) show increases in WNT3A levels (c, d), cytosolic and nuclear levels of $\beta$-catenin $(\mathbf{e}, \mathbf{f})$ and protein levels of WNT target genes $(\mathbf{g}, \mathbf{h})$ in the kidneys of $d b / d b$ mice. GAPDH, glyceraldehyde3-phosphate dehydrogenase. Each lane represents an individual animal. Values are mean $\pm \mathrm{SD}, n=4$; WT, white bars; $d b / d b$ mice, black bars; ${ }^{*} p<0.05$ and $* * p<0.01$
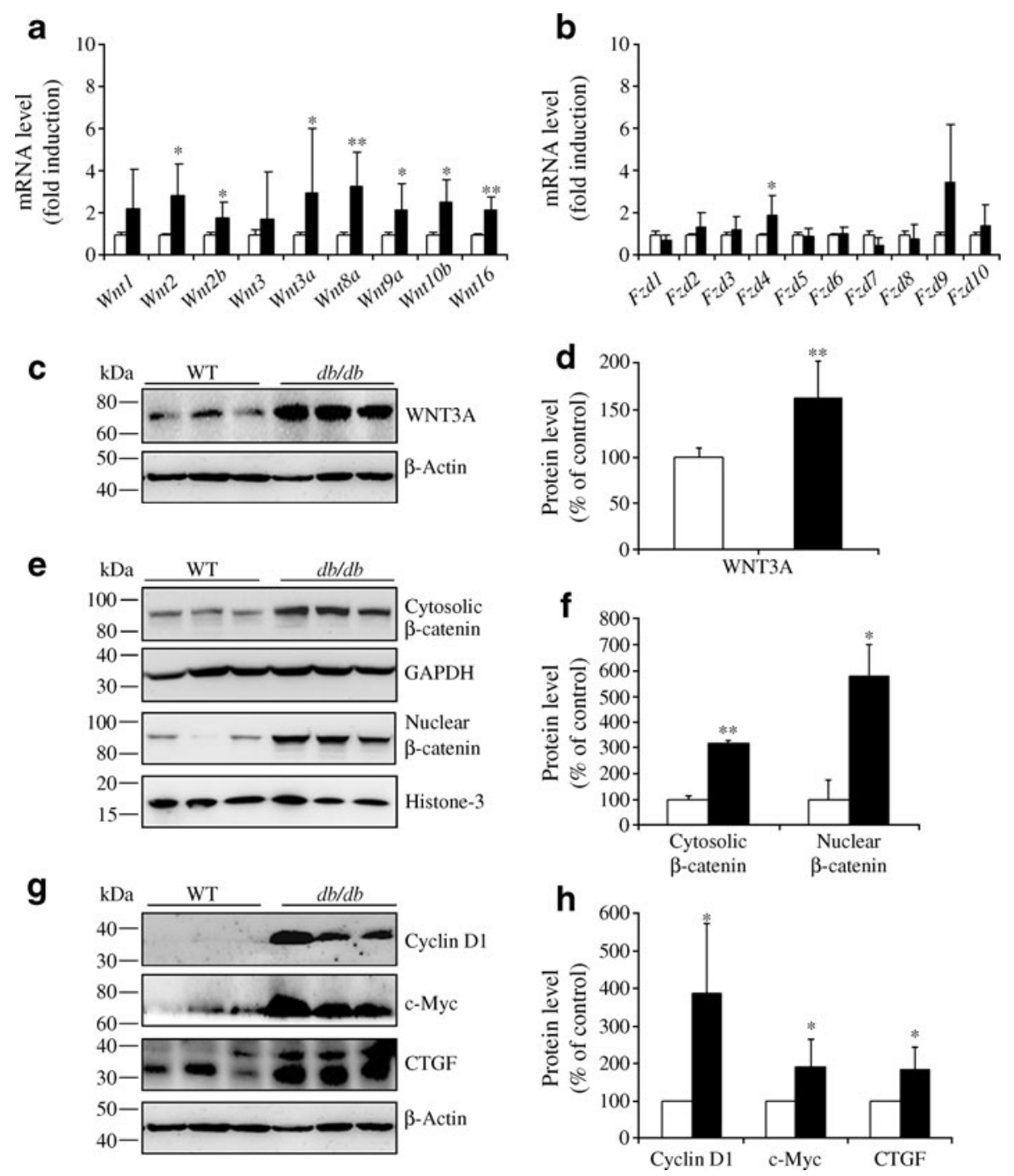

attenuated by $2 \mathrm{~F} 1$ compared with the Akita mice injected with non-specific IgG. Consistent with the decreased $\beta$-catenin levels, the level of CTGF was reduced in the Akita kidney by 2F1 (Fig. 9a, b). Moreover, the production of fibronectin was increased in the Akita kidneys and decreased by 2F1 (Fig. 9c). Masson's Trichrome stain of Akita renal sections showed larger glomeruli with a mildly increased mesangial component and focal blue colouring compared with those of the WT mice; 2F1 partially reversed collagen accumulation (Fig. 9d). These results suggest that the inhibition of WNT signalling ameliorates fibrosis in the diabetic kidney. Functionally, the effect of $2 \mathrm{~F} 1$ on diabetic nephropathy was evaluated by measuring albumin concentrations in $24 \mathrm{~h}$ urine and normalising by creatinine levels. The Akita mice treated with $2 \mathrm{~F} 1$ showed a significant decrease in albuminuria compared with the IgG-treated group (Fig. 9e), suggesting that blockage of the WNT pathway has beneficial effects on diabetic nephropathy.

\section{Discussion}

The present study reports a novel systematic evaluation of the expression of genes encoding components of the WNT signalling pathway in the kidneys of animal models of both type 1 and type 2 diabetes. The results show that WNT signalling is overactivated in the kidneys of models of both type 1 and type 2 diabetes. In addition, hyperglycaemia and oxidative stress were found to play causative roles in the WNT pathway activation in the kidney of diabetic animals. Moreover, blockage of WNT signalling by a monoclonal antibody to LRP6 ameliorated diabetic nephropathy. These results suggest that dysregulation of the WNT pathway in the diabetic kidney plays a pathogenic role in diabetic nephropathy.

We have determined the expression levels of all known WNT genes encoding WNT ligands involved in the canonical WNT pathway. One interesting observation is that there is concurrent upregulation of multiple WNT ligands. Although different diabetic animal models show 


\section{a}
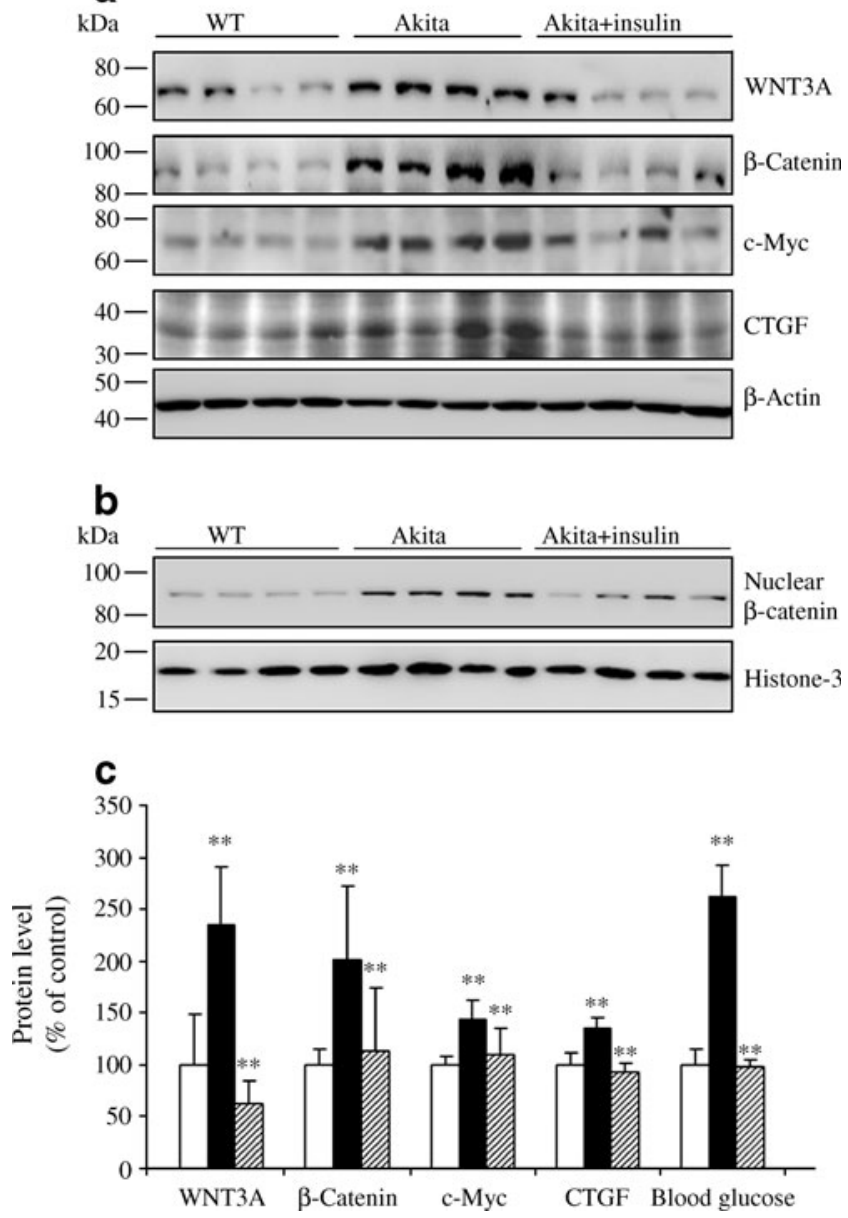

Fig. 5 Effect of hyperglycaemia on the activation of the canonical WNT pathway in the kidneys of Akita mice. Akita mice at the age of 12 weeks were implanted with subcutaneous insulin pellets which provided sustained insulin release for 8 weeks. (a, b) Representative western blot analysis of WNT3A, $\beta$-catenin and WNT target gene products in the kidneys of WT mice, Akita mice and Akita mice with insulin treatment. Whole-kidney homogenates (a) and nuclear proteins (b) were used for western blot analysis. Each lane represents an individual animal. c Blood glucose levels and levels of WNT3A, $\beta$-catenin, c-Myc and CTGF. Values are mean $\pm \mathrm{SD}, n=4$; WT, white bars; Akita mice, black bars; Akita mice with insulin treatment, hatched bars; $* * p<0.01$

different profiles of WNT ligand upregulation, none of the canonical WNT ligands analysed was found to be downregulated in the kidney of any of the diabetic models. This suggests that most WNT ligands are positively regulated in the kidney by diabetes. Of note, the mRNA expression of $W n t 3 a$ and $W n t 10 b$ in the kidney was upregulated in both the type 1 (Akita mice and STZ-induced diabetic rats) and type $2(d b / d b$ mice $)$ diabetic models, indicating that induction of Wnt $3 a$ and $W n t 10 b$ expression is common in diabetic kidneys. Compared with the STZ-induced diabetic rats, Akita mice showed upregulation of more WNT ligands. In parallel, it has been reported that Akita mice develop more severe diabetic nephropathy compared with STZ-treated animals [29]. However, it has been reported that overexpression of Wnt $10 \mathrm{~b}$ was accompanied by a reduction of plasma levels of triacylglycerol as well as improved glucose homeostasis [31]. This disparity suggests that local $W n t 10 b$ regulation in the kidney is different from its systemic regulation.

Moreover, there are apparent differences in WNT ligand and receptor gene expression between the three rodent models. We speculated that the differences may be due to differences in species, genetic backgrounds and ages of the animal models. Different severities and durations of diabetes may also contribute to the differences in WNT ligand upregulation. In addition to diabetic nephropathy, obstructive kidney injury and ischaemia-reperfusion injury have also a

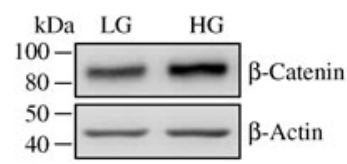

b

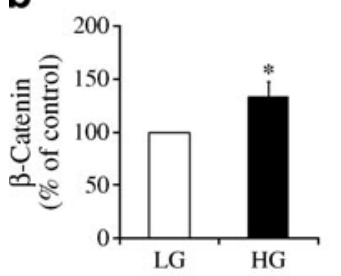

e

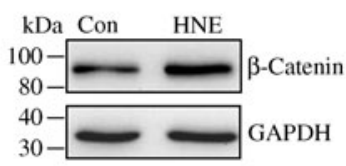

f

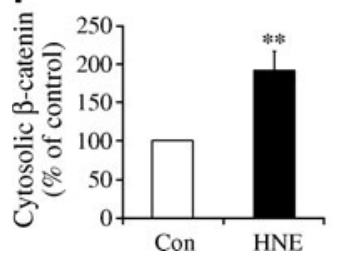

C

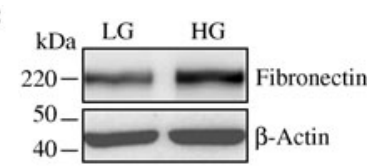

d

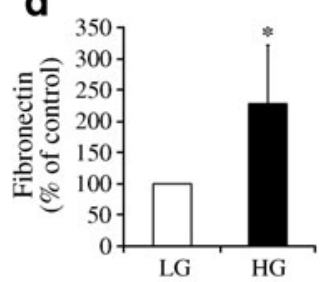

g

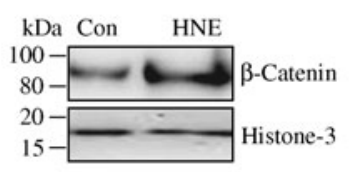

h

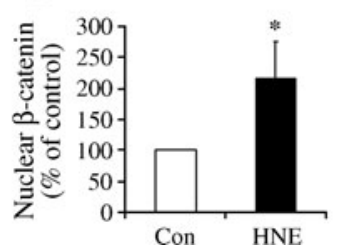

Fig. 6 Activation of the WNT pathway by high glucose and HNE in HRPTC. a-d HRPTC were treated with low-glucose medium (LG, $5 \mathrm{mmol} / \mathrm{l} \mathrm{D}$-glucose and $25 \mathrm{mmol} / \mathrm{l} \mathrm{L}$-glucose) and high-glucose medium (HG, $30 \mathrm{mmol} / \mathrm{l})$ for $12 \mathrm{~h} \mathrm{(a,} \mathrm{b)} \mathrm{and} 24 \mathrm{~h} \mathrm{(c,} \mathrm{d).} \mathrm{Cytosolic}$ $\beta$-catenin and fibronectin levels were determined by western blot analysis, quantified by densitometry from three independent experiments and normalised to $\beta$-actin levels. Values are expressed as the percentages of the control (Con) values (mean $\pm \mathrm{SD}, n=3$ ); ${ }^{*} p<0.05$. e-h HRPTC were treated with HNE $(10 \mu \mathrm{mol} / \mathrm{l})$ for $6 \mathrm{~h}$. Cytosolic $(\mathbf{e}, \mathbf{f})$ and nuclear $(\mathbf{g}, \mathbf{h}) \beta$-catenin levels were measured by western blot analysis using the cytosolic and nuclear fractions, quantified by densitometry from three independent experiments, and normalised to glyceraldehyde-3-phosphate dehydrogenase (GAPDH) levels or histone-3 levels, respectively. Values are expressed as percentages of control values (mean $\pm \mathrm{SD}, n=3$ ); ${ }^{*} p<0.05$ and $* * p<0.01$ 

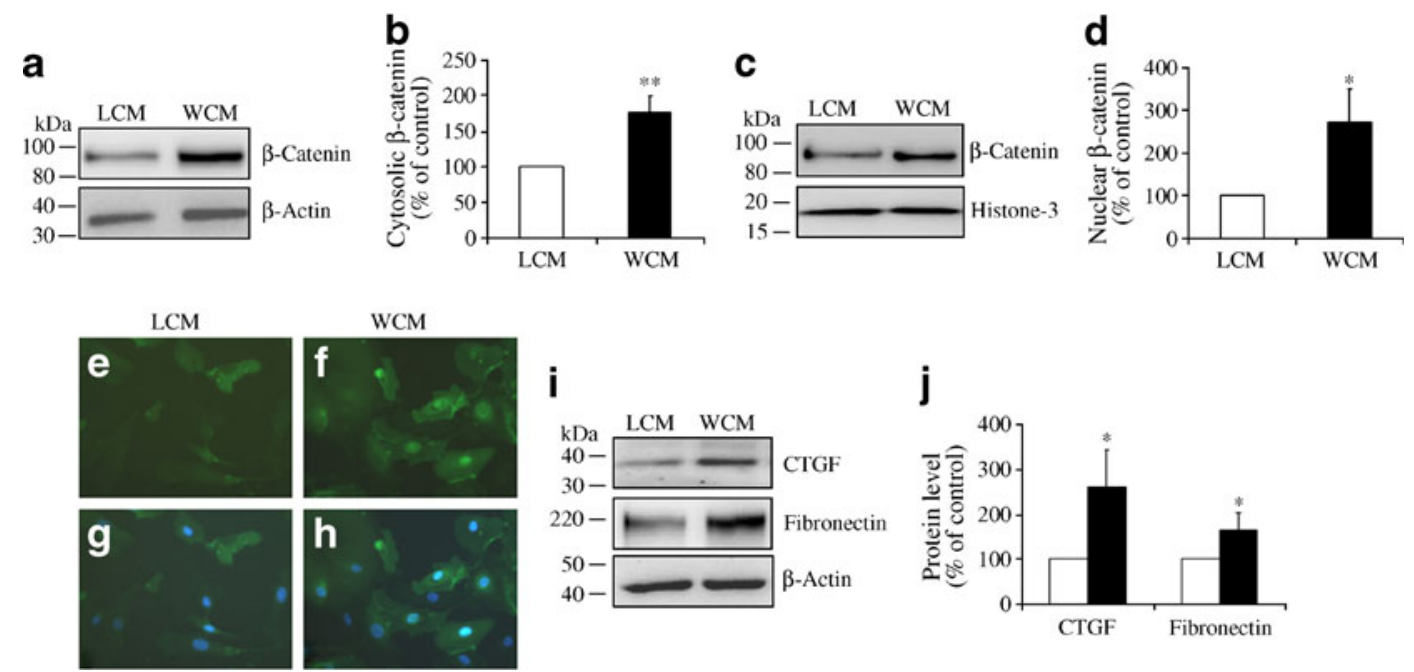

Fig. 7 Upregulation of pro-fibrogenic factors in HRPTC by WNT3A. a-d HRPTC were treated with $50 \%$ vol./vol. LCM or WCM for $12 \mathrm{~h}$. Levels of cytosolic $\beta$-catenin (a, b) and nuclear $\beta$-catenin $(\mathbf{c}, \mathbf{d})$ were determined by Western blot analysis, quantified by densitometry from three independent experiments and normalised to GAPDH levels or histone-3 levels, respectively. Values are expressed as percentages of those in the LCM controls (mean $\pm \mathrm{SD}, n=3$ ); ${ }^{*} p<0.05$ and ${ }^{* *} p<0.01$. e-h Immunostaining of the (e, g) LCM- and (f, h) WCM-treated cells using an antibody specific for $\beta$-catenin revealed the subcellular

been shown to induce overexpression of Wnt1, Wnt2, Wnt2b, Wnt3, Wnt3a, Wnt8a, Wnt16, Fzd3, Fzd4, Fzd9 and Fzd10 gene expression in kidney $[14,25]$, suggesting that the activation of the WNT signalling pathway may be a common pathogenic mechanism for some kidney diseases.
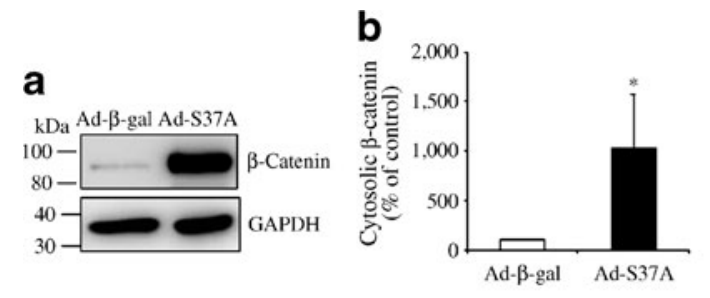
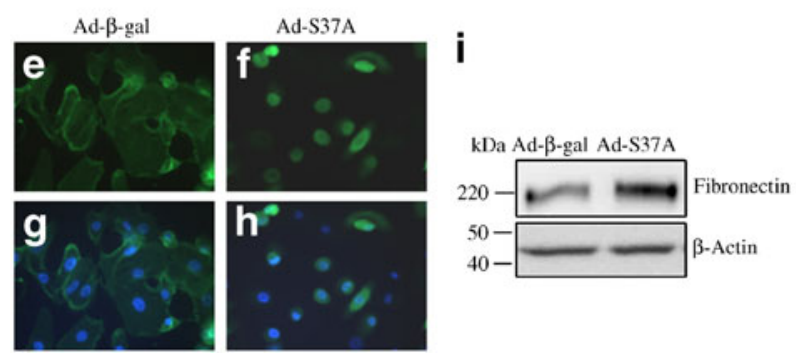

localisation of $\beta$-catenin. e, $\mathbf{f} \beta$-catenin signal (green); (g, h) $\beta$-catenin signal merged with DAPI staining of the nucleus (blue). $\mathbf{i}, \mathbf{j}$ The cells were treated with $50 \%$ vol./vol. LCM and WCM for $24 \mathrm{~h}$. Cellular levels of CTGF and fibronectin were determined by western blot analysis, quantified by densitometry from three independent experiments, normalised by $\beta$-actin levels and expressed as percentages of those in the cells treated with $\mathrm{LCM}(\operatorname{mean} \pm \mathrm{SD}, n=3)$; LCM, white bars; WCM, black bars; * $p<0.05$

It has been well documented that good glycaemic control reduces the risk or arrests the progression of diabetic complications $[32,33]$. The present study showed that levels of blood glucose negatively correlated with renal WNT signalling activities in Akita mice treated with insulin. This

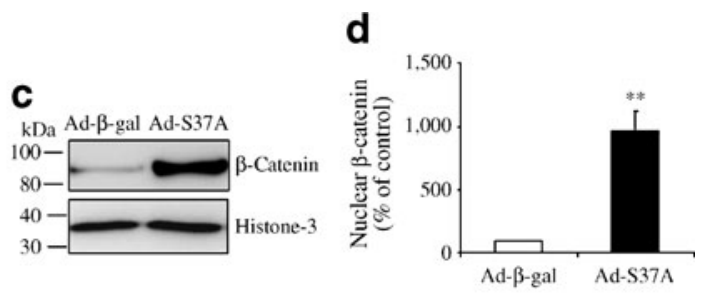

Fig. 8 Induction of fibronectin production in HRPTC by a constitutively active mutant of $\beta$-catenin. a-d HRPTC were infected with Ad$\beta$-gal or Ad-S37A at the same MOI for $48 \mathrm{~h}$. Cytosolic $\beta$-catenin $(\mathbf{a}, \mathbf{b})$ and nuclear $\beta$-catenin $(\mathbf{c}, \mathbf{d})$ were measured by western blot analysis and quantified by densitometry, normalised to glyceraldehyde-3-phosphate dehydrogenase (GAPDH) levels or histone-3 levels, respectively, and averaged in three independent experiments. Values are expressed as percentages of those in Ad- $\beta$-gal control (mean $\pm \mathrm{SD}$, $n=3) ;{ }^{*} p<0.05$ and ${ }^{*} p<<0.01$. $\mathbf{e}-\mathbf{h}$ Immunostaining of the $(\mathbf{e}, \mathbf{g})$ Ad- $\beta$ - gal- and (f, h) Ad-S37A-infected cells using an antibody specific for $\beta$ catenin revealed the subcellular localisation of $\beta$-catenin. $\mathbf{e}, \mathbf{f} \beta$-catenin signal; g, h $\beta$-catenin signal merged with DAPI staining. i Cellular levels of fibronectin were determined by western blot analysis. $\mathbf{j}$ Fibronectin levels were quantified by densitometry from three independent experiments, normalised by $\beta$-actin levels and expressed as percentages of those in the Ad- $\beta$-gal control (mean $\pm \mathrm{SD}, n=3$ ); $* * p<0.01$ 
a

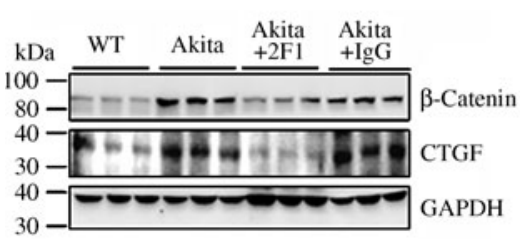

b

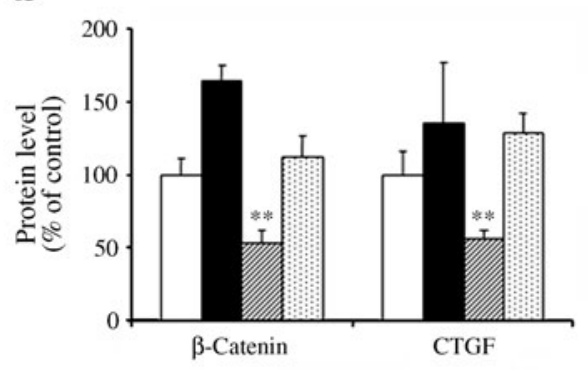

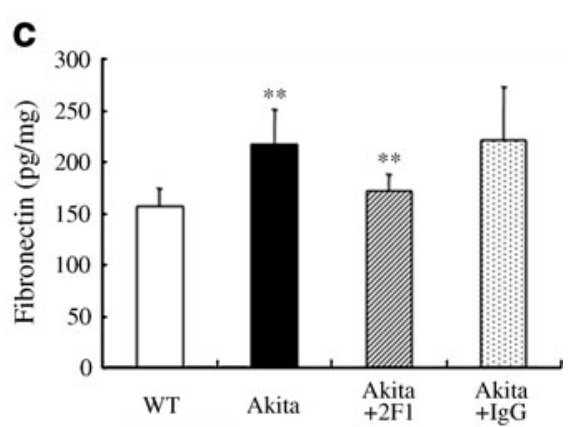

d

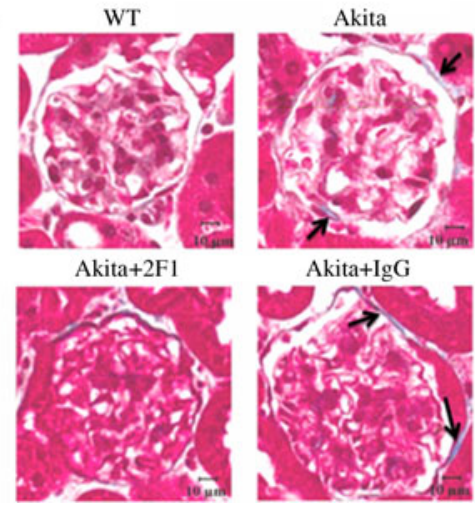

e

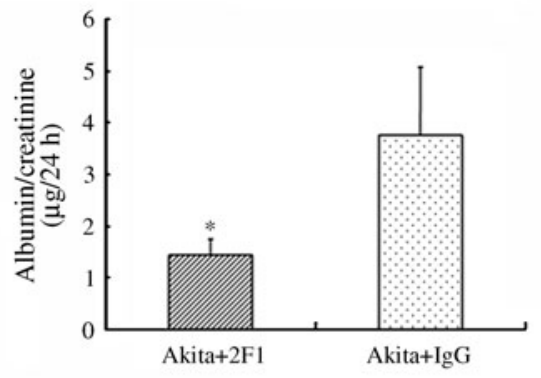

with control treatment, dotted bars; ${ }^{* *} p<0.01$ vs untreated Akita mice. c Fibronectin levels in the kidney were measured using ELISA, normalised by total protein concentrations and expressed as $\mathrm{pg} / \mathrm{mg}$ kidney protein (mean $\pm \mathrm{SD}, n=4$ ). d Paraffin-embedded kidney sections were stained with Masson's Trichrome for tissue collagen. e At the end of the $2 \mathrm{~F} 1$ treatment, $24 \mathrm{~h}$ urine was collected and albumin and creatinine concentrations were measured by ELISA and HPLC, respectively. Values are mean $\pm \mathrm{SD}, n=5$; Akita mice with $2 \mathrm{~F} 1$ treatment, hatched bars; Akita mice with control treatment, dotted bars; $* p<0.05$ compared with Akita mice treated with control $\mathrm{IgG}$

result suggests a causative role of hyperglycaemia in WNT pathway activation in diabetic nephropathy. This notion is supported by the observation that high-glucose medium induces WNT signalling in cultured renal tubular epithelial cells. Reactive oxygen species are considered the common denominator and amplifier of the cellular pathway activated by hyperglycaemia [34-36]. Our previous study also showed that oxidative stress is responsible for WNT pathway activation in cultured retinal cells and in the retina of diabetic rats [18]. Consistent with this, the present study showed that HNE activated the WNT pathway in renal tubular epithelial cells, suggesting that oxidative stress in diabetes also contributes to WNT pathway activation in the diabetic kidney.

The canonical WNT signalling pathway is known to mediate inflammation, angiogenesis and fibrosis through upregulation of intercellular adhesion molecule 1 (ICAM-1), TNF- $\alpha$, VEGF and CTGF, which are all products of WNT target genes [10, 18]. It has been reported that WNT1 protein levels are increased in the podocytes of human kidney biopsies from patients with diabetic nephropathy [16]. In addition, expression of $M M P 7$, a WNT target gene, was strongly increased in the tubular epithelial cells in human kidneys with diabetic nephropathy [37]. Expression of osteopontin, another WNT target gene, was induced in proximal tubular cells of diabetic rats [38]. Compared with other cell types in the kidneys, proximal tubular cells are exposed to conditions of elevated glucose concentrations both apically, as a result of glycosuria, and basally, as a result of elevated interstitial tissue concentrations of glucose [39]. In addition, they express most of the FZD receptors [14] and are the major WNT target cells [25]. Tubular epithelial cells are the predominant cell type in the normal renal interstitium. They not only produce various profibrogenic factors in response to high glucose and increased reactive oxygen species, but also serve as a target for cytokines and relay fibrogenic signals to cortical fibroblasts in the diseased kidney [5].

It is postulated that activated proximal tubular cells indirectly contribute to interstitial fibrosis by activation of 
interstitial fibroblasts. In turn, these stimulated fibroblasts amplify this response by their reciprocal action on the proximal tubular epithelial cells [5]. In diabetic nephropathy, the expression of mesenchymal markers in tubular epithelial cells is well correlated with declining renal function [40]. It is evident that macrophages could be a source of the WNT ligands to which epithelial cells respond in ischaemiareperfusion injury [25]. It was recently reported that WNT/ $\beta$-catenin signalling promotes renal interstitial fibrosis after obstructive injury [14]. However, the implication of WNT signalling in interstitial fibrosis in diabetic nephropathy is largely unknown. Here, we first reported that activation of WNT signalling alone without high glucose can induce the production of CTGF and fibronectin, which are key players in fibrosis in diabetic nephropathy $[1,6,41]$. These results render new evidence to emphasise the importance of WNT signalling and tubular cells in the fibrosis associated with diabetic nephropathy.

WNT signalling initiates when a WNT ligand binds to FZD and LRP5/6 co-receptors [9]. As there are multiple WNT ligands and FZD receptors, but only two co-receptorsLRP5 and LRP6 - we used an antibody specific for the ligand-binding domain of LRP6 to inhibit WNT signalling [30]. Injection of this antibody into Akita mice inhibited the activation of the WNT pathway and ameliorated diabetic nephropathy as shown by fibrogenic factor production, extracellular matrix accumulation and proteinuria. Meanwhile, delivery of serine (or cysteine) peptidase inhibitor, clade A, member 3K (SERPINA3K), an endogenous antagonist of LRP6 [42], also reduced albuminuria in $d b / d b$ mice (Electronic supplementary material [ESM] Fig. 1). These observations indicate a pathogenic role for WNT pathway activation in diabetic nephropathy.

Moreover, our study also suggests that targeting WNT signalling is a promising strategy to hinder the progression of diabetic nephropathy. It is emerging that anti-TGF- $\beta$ antibody and a CTGF antisense oligonucleotide ameliorate renal fibrosis [43, 44]. Our results suggest that an antagonist of WNT signalling may have advantages over those treatments targeting individual growth factors, because WNT signalling regulates the production of a spectrum of pathogenic factors of diabetic retinopathy, including VEGF, CTGF, ICAM-1, TNF- $\alpha$, Twist homologue 1 (TWIST) and matrix metalloproteinase $7[10,14,37$, 45]. This notion is substantiated by previous reports demonstrating that dickkopf homologue 1 (DKK1) and secreted frizzled-related protein 4 (SFRP4) were able to ameliorate renal fibrosis $[14,46]$.

In conclusion, our study demonstrates dysregulation of the WNT signalling pathway in diabetic nephropathy and establishes oxidative stress and hyperglycaemia as activators of the canonical WNT pathway in the kidneys of diabetic rodent models. Furthermore, blocking WNT signalling ameliorated the renal dysfunction. Therefore, our observation underscores the pivotal role of the canonical WNT pathway in the pathogenesis of diabetic nephropathy.

Acknowledgements This study was supported by: National Institutes of Health Grants EY018659, EY012231, EY019309 and P20RR024215; and a research award from the American Diabetes Association.

Contribution statement All authors had substantial contribution to: conception and design, or analysis and interpretation of data; drafting the article or revising it critically for important intellectual content; and final approval of the version to be published.

Duality of interest The authors declare that there is no duality of interest associated with this manuscript.

\section{References}

1. Chiarelli F, Gaspari S, Marcovecchio ML (2009) Role of growth factors in diabetic kidney disease. Horm Metab Res 41:585-593

2. Fioretto P, Mauer M (2007) Histopathology of diabetic nephropathy. Semin Nephrol 27:195-207

3. Kanwar YS, Wada J, Sun L et al (2008) Diabetic nephropathy: mechanisms of renal disease progression. Exp Biol Med (Maywood) 233:4-11

4. Nangaku M (2004) Mechanisms of tubulointerstitial injury in the kidney: final common pathways to end-stage renal failure. Intern Med 43:9-17

5. Phillips AO, Steadman R (2002) Diabetic nephropathy: the central role of renal proximal tubular cells in tubulointerstitial injury. Histol Histopathol 17:247-252

6. Ban CR, Twigg SM (2008) Fibrosis in diabetes complications: pathogenic mechanisms and circulating and urinary markers. Vasc Health Risk Manag 4:575-596

7. Wodarz A, Nusse R (1998) Mechanisms of Wnt signalling in development. Annu Rev Cell Dev Biol 14:59-88

8. Liu C, Li Y, Semenov M et al (2002) Control of beta-catenin phosphorylation/degradation by a dual-kinase mechanism. Cell 108:837-847

9. He X, Semenov M, Tamai K, Zeng X (2004) LDL receptor-related proteins 5 and 6 in Wnt/beta-catenin signalling: arrows point the way. Development 131:1663-1677

10. Si W, Kang Q, Luu HH et al (2006) CCN1/Cyr61 is regulated by the canonical Wnt signal and plays an important role in Wnt3Ainduced osteoblast differentiation of mesenchymal stem cells. Mol Cell Biol 26:2955-2964

11. Chen Y, Hu Y, Zhou T et al (2009) Activation of the Wnt pathway plays a pathogenic role in diabetic retinopathy in humans and animal models. Am J Pathol 175:2676-2685

12. Romero-Aroca P, Mendez-Marin I, Baget-Bernaldiz M, Fernendez-Ballart J, Santos-Blanco E (2010) Review of the relationship between renal and retinal microangiopathy in diabetes mellitus patients. Curr Diabetes Rev 6:88-101

13. Pulkkinen K, Murugan S, Vainio S (2008) Wnt signalling in kidney development and disease. Organogenesis 4:55-59

14. He W, Dai C, Li Y, Zeng G, Monga SP, Liu Y (2009) Wnt/beta-catenin signalling promotes renal interstitial fibrosis. J Am Soc Nephrol 20:765-776

15. Lancaster MA, Gleeson JG (2010) Cystic kidney disease: the role of Wnt signalling. Trends Mol Med 16:349-360 
16. Dai C, Stolz DB, Kiss LP, Monga SP, Holzman LB, Liu Y (2009) Wnt/beta-catenin signalling promotes podocyte dysfunction and albuminuria. J Am Soc Nephrol 20:1997-2008

17. Wang JJ, Zhang SX, Mott R et al (2006) Salutary effect of pigment epithelium-derived factor in diabetic nephropathy: evidence for antifibrogenic activities. Diabetes 55:1678-1685

18. Zhou T, Hu Y, Chen Y et al (2010) The pathogenic role of the canonical Wnt pathway in age-related macular degeneration. Invest Ophthalmol Vis Sci 51:4371-4379

19. Dunn SR, Qi Z, Bottinger EP, Breyer MD, Sharma K (2004) Utility of endogenous creatinine clearance as a measure of renal function in mice. Kidney Int 65:1959-1967

20. Gurley SB, Mach CL, Stegbauer J et al (2010) Influence of genetic background on albuminuria and kidney injury in Ins2(+/ C96Y) (Akita) mice. Am J Physiol Renal Physiol 298:F788-F795

21. Schrijvers BF, de Vriese AS, Flyvbjerg A (2004) From hyperglycemia to diabetic kidney disease: the role of metabolic, hemodynamic, intracellular factors and growth factors/cytokines. Endocr Rev 25:971-1010

22. Huang JS, Chuang LY, Guh JY, Huang YJ, Hsu MS (2007) Antioxidants attenuate high glucose-induced hypertrophic growth in renal tubular epithelial cells. Am J Physiol Renal Physiol 293: F1072-F1082

23. Calabrese V, Mancuso C, Sapienza M et al (2007) Oxidative stress and cellular stress response in diabetic nephropathy. Cell Stress Chaperones 12:299-306

24. Furfaro AL, Menini S, Patriarca S et al (2005) HNE-dependent molecular damage in diabetic nephropathy and its possible prevention by $N$-acetyl-cysteine and oxerutin. Biofactors 24:291-298

25. Lin SL, Li B, Rao S et al (2010) Macrophage Wnt7b is critical for kidney repair and regeneration. Proc Natl Acad Sci USA 107:4194-4199

26. Rane MJ, Song Y, Jin S et al (2010) Interplay between Akt and p38 MAPK pathways in the regulation of renal tubular cell apoptosis associated with diabetic nephropathy. Am J Physiol Renal Physiol 298:F49-F61

27. Ortiz-Munoz G, Lopez-Parra V, Lopez-Franco O et al (2010) Suppressors of cytokine signalling abrogate diabetic nephropathy. J Am Soc Nephrol 21:763-772

28. Okazaki Y, Yamasaki Y, Uchida HA et al (2007) Enhanced TGFbeta/Smad signalling in the early stage of diabetic nephropathy is independent of the AT1a receptor. Clin Exp Nephrol 11:77-87

29. Poli G, Schaur RJ, Siems WG, Leonarduzzi G (2008) 4-Hydroxynonenal: a membrane lipid oxidation product of medicinal interest. Med Res Rev 28:569-631

30. Zhou T, Zhou KK, Lee K et al (2010) The role of lipid peroxidation products and oxidative stress in activation of the canonical wingless-type MMTV integration site (WNT) pathway in a rat model of diabetic retinopathy. Diabetologia 54:459-468

31. Aslanidi G, Kroutov V, Philipsberg G et al (2007) Ectopic expression of Wnt10b decreases adiposity and improves glucose homeostasis in obese rats. Am J Physiol Endocrinol Metab 293: E726-E736

32. Vasudevan AR, Burns A, Fonseca VA (2006) The effectiveness of intensive glycemic control for the prevention of vascular complications in diabetes mellitus. Treat Endocrinol $5: 273-286$

33. Eastman DK, Bottenberg MM, Hegge KA, Ourth H, Kabadi U (2009) Intensive insulin therapy in critical care settings. Curr Clin Pharmacol 4:71-77

34. Hakim FA, Pflueger A (2010) Role of oxidative stress in diabetic kidney disease. Med Sci Monit 16:RA37-RA48

35. Ha H, Hwang IA, Park JH, Lee HB (2008) Role of reactive oxygen species in the pathogenesis of diabetic nephropathy. Diabetes Res Clin Pract 82(Suppl 1):S42-S45

36. Wagener FA, Dekker D, Berden JH, Scharstuhl A, van der Vlag J (2009) The role of reactive oxygen species in apoptosis of the diabetic kidney. Apoptosis 14:1451-1458

37. Cohen CD, Lindenmeyer MT, Eichinger F et al (2008) Improved elucidation of biological processes linked to diabetic nephropathy by single probe-based microarray data analysis. PLoS One 3: e2937

38. Hsieh TJ, Chen R, Zhang SL et al (2006) Upregulation of osteopontin gene expression in diabetic rat proximal tubular cells revealed by microarray profiling. Kidney Int 69:1005-1015

39. Morrisey K, Steadman R, Williams JD, Phillips AO (1999) Renal proximal tubular cell fibronectin accumulation in response to glucose is polyol pathway dependent. Kidney Int 55:160-167

40. Liu Y (2009) New insights into epithelial-mesenchymal transition in kidney fibrosis. J Am Soc Nephrol 21:212-222

41. Chen CC, Lau LF (2009) Functions and mechanisms of action of CCN matricellular proteins. Int J Biochem Cell Biol 41:771-783

42. Zhang B, Abreu JG, Zhou K et al (2010) Blocking the Wnt pathway, a unifying mechanism for an angiogenic inhibitor in the serine proteinase inhibitor family. Proc Natl Acad Sci USA 107:6900-6905

43. Ziyadeh FN, Hoffman BB, Han DC et al (2000) Long-term prevention of renal insufficiency, excess matrix gene expression, and glomerular mesangial matrix expansion by treatment with monoclonal antitransforming growth factor-beta antibody in $\mathrm{db} / \mathrm{db}$ diabetic mice. Proc Natl Acad Sci USA 97:8015-8020

44. Guha M, Xu ZG, Tung D, Lanting L, Natarajan R (2007) Specific down-regulation of connective tissue growth factor attenuates progression of nephropathy in mouse models of type 1 and type 2 diabetes. FASEB J 21:3355-3368

45. Elmarakby AA, Sullivan JC (2011) Relationship between oxidative stress and inflammatory cytokines in diabetic nephropathy. Cardiovasc Ther. doi:10.1111/j.1755-5922.2010.00218.x

46. Surendran K, Schiavi S, Hruska KA (2005) Wnt-dependent beta-catenin signalling is activated after unilateral ureteral obstruction, and recombinant secreted frizzled-related protein 4 alters the progression of renal fibrosis. J Am Soc Nephrol 16:2373-2384 